Portland State University

PDXScholar

\title{
The Role of Translational Neuroscience in the Evidence-Based Practice of Clinical Psychology
}

\author{
Anthony Beers \\ Portland State University
}

Follow this and additional works at: https://pdxscholar.library.pdx.edu/honorstheses Let us know how access to this document benefits you.

\section{Recommended Citation}

Beers, Anthony, "The Role of Translational Neuroscience in the Evidence-Based Practice of Clinical Psychology" (2014). University Honors Theses. Paper 164.

https://doi.org/10.15760/honors.109

This Thesis is brought to you for free and open access. It has been accepted for inclusion in University Honors Theses by an authorized administrator of PDXScholar. For more information, please contact pdxscholar@pdx.edu. 


\title{
The Role of Translational Neuroscience in the Evidence- Based Practice of Clinical Psychology
}

\author{
by \\ Anthony Beers
}

An undergraduate honors thesis submitted in partial fulfillment of the

requirements for the degree of

Bachelor of Science

in

University Honors

and

Psychology \& Science

Thesis Adviser

Dr. Dennis Swiercinsky

Portland State University

2014 


\section{Abstract}

Is the Information Age progressing to the Knowledge Overload Age? The presence of big data, complex systems, and vast knowledge networks can overwhelm a clinician's capacity to sift through abundant research findings and theoretical models. Demand for economically driven evidence-based practice and the ethics of incorporating continuing education place enormous demands on clinicians that are difficult to achieve effectively, efficiently, and ethically. Translational science is a research discipline focused specifically on the production of valid and reliable uses developed from scientific research findings. In the discussions on translational science, one controversial issue has been the role translational neuroscience plays in clinical psychology. On the one hand, translational neuroscience has been argued as a research discipline bridging scientific discoveries of the brain and the creation of novel standardized psychological treatments. On the other hand, some describe translational neuroscience as the clinician's daily use of current scientific understanding in the clinical setting. The purpose of this study is to demonstrate that clinical psychologists are actively translating neuroscience research into clinical practice and to emphasize the need for the development of tools and support structures that facilitate this process at the clinical level. This thesis focuses on a summary of the types of neuroscience research and research disciplines influencing the practice of clinical psychology, case studies demonstrating current translation of neuroscience into clinical practice and the ethical implications of this ongoing translational process of neuroscience research into the clinical setting. 


\section{Challenge of Clinical Practice}

\section{Understanding Human Behavior}

A psychologist's primary task is to actively observe and interpret human behavior via a synthesis of patient-communicated and directly observed patient responses. The integration of graduate training with professional on-the-job experience generates the expert capacity to diagnose and treat psychological disorders. This is a continual process for professionals who work their entire careers to establish and maintain a personal standard-of-care that will hold up to scrutiny from professional governing bodies. These governing bodies work to maintain broad professional effectiveness and integrity. In 2005 a mandate by the APA Council of Representatives requires psychological treatments to be supported by empirically based scientific research (American Psychological Association, 2006). The evidence-based principles have their own methodological and implementation challenges but ideally bring greater confidence to both patients and clinicians alike when considering the effectiveness of chosen treatments and treatment plans.

When determining the correct diagnosis and subsequent treatment for patients, clinical psychologists draw from their professional education and experience with similar cases. At times they may seek the consultation of another professional to strengthen their confidence in their approach or to inform their method with complimentary information. With the development of global populations and interdisciplinary research discoveries the number and types of complimentary understandings are growing. A research discipline that influences psychology on nearly all levels is that of neuroscience. Baseline understandings of both the anatomical structures and functional workings of the brain are becoming a foundational part of understanding psychological phenomenon. Diagnosis and treatment effectiveness have been strongly linked to alterations in the structure and function of patients' brains. With the continual development of neuroscientific understandings of the brain, staying abreast with the developments within the neuroscience field as well as finding practical uses for them in the clinical settings is becoming a central part of the clinical psychologist's day. The use of evidence-based practices is necessary in all aspects of the clinician's job with the continuation of education acting as a central component to maintaining clinical effectiveness. 


\section{Staying Current}

Clinical psychologists are mandated to maintain a continuing education (CE) plan that is in line with the standards set forth by the professional governing body. This is often self-regulated and supported through varying private and public CE providers. However, merely wading through the myriad of options can be a challenge in itself with considerations for the quality, practicality, and certification level of the content in mind. $\mathrm{CE}$ is only the baseline for many licensed clinicians as they are otherwise actively searching for, collecting, and reviewing peer reviewed articles as well as participating in professional conferences (Gyani, Shafran, Myles, \& Rose, 2014). The aim is integration of all this information into cogent principles from which individual professionals can inform their current practice. As developments are published from recent research, the boundaries of effective and ethical practice are modified. What constitutes best, evidence-based practice is under constant revision by the collective efforts of both researchers and clinicians. Staying current with these best practices grows ever more challenging for practicing clinicians as the number of research articles and theories published grows each year (Steen, Casadevall, \& Fang, 2013).

Effective clinical psychologists are expected to keep abreast with the developments in all phases of scientific discovery from basic research, such as the psychological and neurobiological underpinnings of behavior, to efficacy evaluations of current treatment methodologies and theoretical models. Establishing a workflow that incorporates the collection, review, and evaluation of these various levels of discovery is a part of requisite training for professionals. The balance between the volumes of reading considered pertinent for maintaining adequate knowledge of effective diagnosis and treatment while maintaining an economically sound practice appears to be shifting. Increases in the number of discoveries made in neuroscience and other brainbased disciplines have broad implications for clinical psychologists. Clinicians making and maintaining this new balance would benefit from the development and dissemination of clinician-level translational methods enabling their own translation of the most recent discoveries. This ability would both increase the effectiveness of the clinician as well as increase the efficiency of basic science translation into practice. In the next section, distinct phases of scientific discovery are explored as well as several disciplines showcasing promising discoveries for understanding psychological illness and treatment. 


\section{Clarifying the contexts}

\section{Basic Research}

Basic research can be understood as the systematic study of phenomena through experimentation yielding information typically based on a reductionist philosophy. This type of research is often used to help determine fundamental relationships between quantifiable experiences. These experiments and efforts are primarily focused on discovery of information in relation to other information in the scientific canon as opposed to creating direct applications for practicing professionals. The same emphasis is mirrored in basic psychological research. Discoveries made in this phase of research are however informative for the theoretical foundations of understanding psychological disease states and for the development of corrective interventions. These theoretical foundations are created by identifying normal versus abnormal psychological symptoms and the differences in related anatomical, physiological and psychological states. Publications are produced with the intent of challenging and/or refining currently supported psychological theories. The accumulation of supporting evidence for a particular theory generates the confidence necessary for professionals to use the theoretical framework in their explanation of accepted and emerging psychological phenomenon.

Typically basic research has been seen as the breeding ground for novel scientific understanding. It can be said that the results of a critical mass of peer-reviewed publications generate the boundaries of acceptable evidence based practice. These discoveries establish the foundations and frameworks from which everyday applications can be translated. Taking scientific discovery from bench to bedside is the common emphasis for translation focused research labs and institutions of today, but such an emphasis situates the most recent discoveries several years away from application in current clinical practices. The increased need in healthcare for greater clinical capacity or adaptation to new diseases requires an enhanced ability to generate immediate use of the wealth of basic research discovery. Adapting basic research publications with standardized language and centralizing theory modifications would facilitate the active translation of research findings into the clinic by a practicing clinician. Alterations such as these would also stay in line with the primary purpose of basic research publications, that is, growing the body of scientific knowledge. Allowing for effective and ethical alternate paths of clinical application, diverted from the translational bottleneck currently experienced in many disciplines, is a needed shift by the scientific community. Getting researchers and clinicians on the "same page" 
with research findings, through translation-focused standardized publications, would allow for much broader and more immediate impact of current scientific findings.

\section{Applied Research}

Applied research methods are commonly designed to yield documented best practices and standardsof-care. This phase of research is where the fine-tuning of clinical practice is done within the context of supported theoretical frameworks. At any one time there could be several possible treatment approaches for a certain psychological condition and applied experiments are designed to compare the different methods and interventions to determine the level of utility, or futility, for each (Thyer \& Myers, 2011). Progress made in applied psychological research works to establish confidence levels with which practitioners can proceed when looking to utilize the different evidence-based practices available. Currently, this type of research is likely to be the most closely followed by practicing clinicians as they are continually addressing these consideration in their day-to-day activities. Findings on the effectiveness of one treatment versus another can have significant implications for the use or alteration of treatment plans.

Applied research traditionally comes after the formation of a new treatment. The process of discovering, developing, and implementing new treatments however can be long and costly. After the generation of novel treatments, the next concern is communication of that information to the parties in need. Coupling the communication efforts of applied research discoveries with that of related clinician-level translational approaches would bolster the utility of the information resources available. The development of methods for disseminating translation focused knowledge to these clinicians is in need (Shoham et al., 2014). There is an opportunity for the institutions dedicated to applied research to become central hubs for the synthesis and distribution of the information pertinent to clinical practitioners at both the treatment and the translational level. As many of these researchers and institutions are already uniquely focused on the broad communication of effective treatment approaches the addition of clinician-level translation approaches would be integrated well. When bearing in mind the active translation of research into practice by clinicians, having this goal of more effective communication is likely best complimented with collecting and synthesizing information for providers into publicized and open access internet databases. This then provides a resource useable and improvable by the professionals most closely connected to them. 


\section{Translational Research}

Translational research is an emerging, yet somewhat ill-defined, category of research focused on the generation of applications from basic science theories and principles (Woolf SH, 2008). This approach to research has been hotly debated ever since Elias A. Zerhouni wrote his formative article "Translational and Clinical Science — Time for a New Vision” in the New England Journal of Medicine in 2005 (Zerhouni, 2005). Translational science operates to yield knowledge through a synthesis of information from several sources. These sources can include basic and applied research from a variety of disciplines in an effort to create, or augment and adapt, the distinct approaches into a new paradigm. The formalized research in this field is often associated with medical and/or clinical practitioners and the boundaries, utility, ethics, and methodologies of translational science are still in formation for many disciplines (“Translational Psychology," n.d.). For psychology, translational research as a "bridging" research focus is growing with a recent push for emphasis in translational research in both graduate and continuing education across many psychology sub-disciplines (Atkins, Strauman, Cyranowski, \& Kolden, 2014; "Postgrad growth area," n.d.).

Translational research would progress in a different manner with the shift in emphasis of translation onto the clinician. Particularly evident would be the dramatic shift in the number of individuals working on translation of the basic research discoveries into clinical application. Nearly all clinicians, at one point or another, actively translating research into practice could provide many nuanced understandings to a greater translation project. With this shift however, comes the need for the formalization of general translational approaches and the collection and communication of individual approaches. Providing centralized collection would facilitate formalization like this and communication information centers for individual clinicians to collaborate through. This type of standardization would be particularly important when considering the increase in interdisciplinary work in both research and practice. Increasing crossover between scientific disciplines is in many ways providing answers previously unattainable, but knowing the useful intersection of these disciplines is still in formation. Institutions focused on translational research would then be focused on not only their own translational approaches but also providing resources for the collection and integration of individual clinicians' approaches. An approach like this opens up the translational project into a professional crowd source approach. The increase in degrees of complexity with this translational approach would then also require greater attention. Using systems science models and theoretical frameworks for addressing complexity in translating research into 
practice could prove to be the next step towards useful integration while maintaining the integrity of the current clinical and research systems.

\section{Systems Science}

Systems science is a multidisciplinary approach to creating and applying models of understanding which operate through and within several levels of influence on a particular phenomenon via systems or a system of systems perspective. Inherent in systems science's design is the process of reconceptualizing information to yield broad knowledge that leads to consilience. Systems science is a math and logic driven field which is progressing towards further use of data base generation and analysis technologies in an effort to understand complexity. Visuals and computer simulations are commonly produced which could aide in the role systems science plays for knowledge creation and dissemination (Hieronymi, 2013). The visual and explanatory nature of these products of systems science research would prove to be important in efficiently educating professionals and the public on relevant scientific discoveries. Additionally, establishing systems based models that have use for many individuals within an interdisciplinary project would allow for increases in communication and project production enhanced by the centralized vision created within a systems based model.

Some systems science models useable in psychology are already being applied to disciplines such as neuroscience for questions in neural network connections and interactions (Braff L \& Braff DL, 2013). This systems science lens allows for a useful approach to understanding the emergent phenomenon that come from billions of single neuronal cells connected to one another in trillions of different relationships. The field of systems science also has much to offer both researchers and clinicians when it comes to dealing with the issues of big data and mental healthcare (Green, 2006). Understanding what factors most contribute to population health and healthcare service optimization would lead to greater direction of professional efforts as limiting components within the systems could be identified and assessed for improvement needs both immediate and long-term.

For individual clinical psychologists, integration of systems science frameworks would better enable the production and management of relevant scientific publications that influence their evidence-based practice. Increasing useful communication between professionals from research through to clinical application would 
create the most effective translation of basic research to practice. This increase in interconnectivity between fields and professionals however generates another level of complexity for professionals to manage. Approaching this push for interdisciplinary communication would be greatly enhanced by a broad general understanding of systems science frameworks. Full use of systems science in both business and research settings would allow for the optimization of individual roles in the vast and growing scientific system. In the next section, several research disciplines are explored with their relevance to shaping the practice of clinical psychology featured.

\section{Fields Changing the face of Clinical Psychology}

\section{Behavioral and Cognitive Neuroscience}

Behavioral neuroscience (or, referred to as cognitive neuroscience by some) is a field focused on the study of how low level biological processes, particularly in neurons and neuron systems, influence the behavior, cognition, and emotions of humans and non-human organisms. Research in this field focuses on human and nonhuman experimentation emphasizing behavioral processes across genetically similar species. New developments being pursued in the areas of study include language, reasoning, emotions, and consciousness. The discoveries made in these areas inform researchers and clinicians of the biological underpinnings of identifiable behavioral patterns. Many treatments and approaches to remediation can start at the biological level with the intent of altering diseased neurochemical levels towards more physiological normal ones.

Theories and discoveries from behavioral neuroscience have strong implications for clinical psychologists' assessments and treatment plans as discoveries in the biological processes have a bottom up influence on understanding processes in treatment and diagnosis. By understanding the cellular mechanisms and brain chemistry influencing development of diagnosis and treatment plans, clinicians are able to identify disease and target treatments linked to specific biological and neuronal mechanisms. Understanding these areas from a neurobiological level generates a solid bottom-up framework for clinical psychologists when interpreting behaviors and formulating treatment. 


\section{Clinical Psychology and Neuropsychology}

Neuropsychology is a professional field focused on the measurement and diagnosis of brain disorders.

This specialty implements applied science in brain and behavior relationships by using specialized

psychological, neurological, and physiological assessments to inform psychological diagnoses and treatments

(Harvey, 2012). Integration of these assessments can be done in conjunction with work done by other mental

health providers promoting a better understanding of patients and their treatment plans. The growth in the use of neuropsychology assessment is likely due, in part, to an emphasis on quantifiable behavioral, cognitive, and neurological measures which are correlated with brain conditions (Braff L \& Braff DL, 2013). Having quantifiable measures makes neuropsychology a valuable discipline for emphasis when working to establish evidence-based practice in psychology. These quantifiable measures allow for distinctions to be made between healthy and diseased states producing an additional level of analysis when diagnosing and treating patients. Additionally, the standardization of associated disease state measures could produce the capacity to track patterns in neurological adaptation over time.

The ability for clinical psychologists to include these assessments in understanding a patient's brain state allows for neurophysiological contextualization of behaviors. These assessments enhance the psychologist's understanding of the inner workings of their patient's brain. Neurological assessment use has had increasing support for their integration and emphasis in the training of mental health clinicians (Coverdale et al., 2014). Formalized education focused on useful methods of integration of neuropsychological assessments in clinical psychology increases understandings of the neural networks present in clinicians' patients and facilitates the use of quantifiable measures such as brain-biomarkers related to illness and disease. Establishing these measureable and repeatable indicators of neurophysiological states will lead to a greater confidence in the diagnosis and treatment of psychological disorders when used in the clinical setting. Integration of these more global assessments of brain function with the neurobiological understandings generated in behavioral neuroscience research will subsequently add an additional level of confidence to the psychologist's neuroscience informed practice. 


\section{Brain Imaging and the Connectome}

Advancements in brain imaging have allowed for detailed snapshots as well as dynamic time-based analyses to be obtained on both human and nonhuman models. The connectome is an imaging project of intense, recent attention in neuroscience. Many projects have been designed as integrative, international and interdisciplinary approaches to mapping neural connections within the human brain both structurally and functionally (Biswal et al., 2010). Copious amounts of data have and will continue to be produced through projects such as the Human Connectome Project, Brainnetome Project, the CONNECT project and 1000 Functional Connectomes (Assaf et al., 2013; Jiang, 2013; Van Essen et al., 2013; Yan, Craddock, Zuo, Zang, \& Milham, 2013). Databases created from these projects are developing as open access to allow for cross project analyses and increased strength in computational models and simulations. The understanding generated from research of this kind is focused on the integration of the multilevel approaches to understanding human brain function. Collaboration within these fields will allow investigators to tackle complexity and dynamicity present in the development and adaptation of connectivity and function in the brain's neural networks.

Currently the practical utility of these hypothesis-free datasets is low in clinical psychology as they are underdeveloped. However, with continued translational efforts the possible applications are significant. Integration of disease state network modeling and their alteration over the lifespan will lead to a biophysical model richly connected to clinical decision-making needs (Woolrich \& Stephan, 2013). Disease state network models focus on several factors including the indicators developed in clinical practice. Integration of these clinic specific indicators with the microstructural and macro structural analyses available through the databases will intimately tie the understandings of both clinical and research professionals. Disease and treatment specific models will be possible through this collaborative approach and facilitate the generation of useable clinical analyses. Further development of the measurement and modeling technologies as well as integrative professional approaches would lead to the synthesis of measurement methods capable of assisting clinicians with individualized diagnosis and treatment including progress over time. 


\section{Translating Neuroscience into Clinical Practice}

\section{Examples from Current Literature}

Recently there have been increases in the number of publications emphasizing translation and application of various forms of neuroscience research into the clinical practice of psychology. As discussed previously, clinical psychologists are beginning to focus greater attention to the development of brain based understandings of psychological disease and therapy. Three examples of publications demonstrating this focus highlight the use of neuroplasticity measurements, the clinical utility of brain maps and the use of personal monitoring devices for measuring psychological symptoms respectively.

In Clare Kelly and F. Xavier Castellanos' recent article Strengthening Connections: Functional Connectivity and Brain Plasticity published in the Neuropsychology Review this year, the growth in the use of functional neuroimaging techniques is discussed as it relates to neuropsychology. More specifically the use of these neuroimaging techniques and the mapping of macro-scale networks in the human brain to determine changes due to training and practice. In 2004 a review of the literature put forward a framework to guide understanding and interpreting practice on neuroplasticity measured with intrinsic functional connectivity (iFC) focusing on the patterns of induced change through task activation (A. M. C. Kelly, 2004). There are several factors that contribute to the neural changes due to practice and training including: the effect of practice on the cognitive processes supporting task performance, task domain, time-window of imaging, the level of previous experience with the task, individual differences in performance and confounding factors such as task difficulty (C. Kelly \& Castellanos, 2014). Two changes particularly prominent are reorganization and redistribution in task-evoked activity representing changes in cognitive processes and decreased use of front parietal control and attentional processes respectively. When determining the effects of practice and training the period when individuals are imaged is strongly correlated with the shifts in activity observed. This puts an emphasis on the need for capturing the whole of the practice-related effects.

The clinical utility of practice-induced brain plasticity are a focus of Kelly and Castellanos. Developing and refining techniques that measure changes in the human brain, both short term and long term, would allow for a fundamental shift in the treatment of psychological illness and disease. Both medicinal and behavioral approaches to psychological treatment would be informed by the measurement of these changes. 
Enabling measurements of this type with patients over extended periods of time would give psychologists a window in brain based changes relevant to improvement or worsening of patient symptoms and disease states. Translation of neurochemical, neural network and even neuroanatomical understandings relevant to psychological treatment would become uniquely tied. Psychologist, given the ability to monitor brain changes in this way, would also gain the ability to understand the connection between treatments approaches and healthy brain states. Individualized treatment plans would be accessible as the clinician would be able to alter their approach based on each patient behavioral and neural response to treatments. Trends toward healthy brain function from the chemical to the network level would complement the tracking of behavioral symptoms. Castellanos et al. published a 2013 article in Nuroimage, Clinical Applications of the Functional Connectome, focusing on the use of a central neuroimaging database for the discovery and validation of brain based biomarkers useful to clinicians. Emphasis is placed on the need for connecting these resources to clinically related variables as to increase the utility of the findings (C. Kelly, Biswal, Craddock, Castellanos, \& Milham, 2012). Biomarkers discovered through R-fMRI are a keystone in the development of clinical applications as they will act as common ground for both researchers and clinicians to develop translational approaches. The NIH Biomarkers Definitions Working Group defined a biomarker as "a characteristic that is objectively measured and evaluated as an indicator of normal biological processes, pathogenic processes, or pharmacologic responses to a therapeutic intervention" (Atkinson et al., 2001). These biomarkers are associative however and will need to be considered as not having direct biological meaning. Finding significant associations between biomarker features and clinical variables does not necessarily mean discovery of useful clinical biomarkers (Castellanos, Di Martino, Craddock, Mehta, \& Milham, 2013). Clinically useable tests need to meet a minimum on validity, reliability, sensitivity and specificity measures. Validity and reliability can be explored independently of disease state (commonly in the lab with repeated measures) while sensitivity and specificity are tied to the intended use (necessitating tissue, nonhuman or human tissue-based studies). The importance of these measures is particularly relevant if biomarkers are to be used with clinical tools for screening, diagnosis or monitoring disease.

Development and use of biomarkers in the clinical setting would further compliment the neuroplasticity measurements discussed previously. A set of biomarkers specifically referencing neural networks and neuroanatomical structures would developed from the use of neuropsychological assessments. 
Additional accepted brain biomarkers would provide clinical psychologists with a repeatable and scalable tool useful when determining the diagnosis and treatment of patients. These neuroscience-focused indicators would facilitate the translation of neuroscience into the psychologist's practice by providing repeatable brain biomarkers that could be connected with psychological symptoms. With confidence in the clinical relevance of the biomarkers is established the alterations and grouping of these biomarkers then becomes a medium for translational neuroscience to take place. Clinically available measurements and tools like this are a central part of the clinician-focused translation of neuroscience.

Alexander Markowetz et al published an article Psycho-Informatics: Big Data Shaping Modern Psychometrics in Medical Hypotheses this year. In it they discuss measuring emotion, behavior, and cognition and their relationship to the collection and analysis of big data. Understanding these measures are fundamental to mental healthcare practitioners. Specially trained experts typically carry out these measures for research or clinical application by conducting interviews and/or observations. Markowetz et all emphasize that approaches of this type however, can hold back the progress of research findings or clinical interventions as they present an inability for continual, unbiased, noninvasive investigation when data collection requires temporal snapshots with the presence of a specialized professional. Self-report also poses its own difficulties with the validity and reliability necessary to produce useable data as patients' memory and training effects have the potential to alter their responses. Reports from patients have a the tendency towards bias due to the nature of recollection, alterations in response influenced by present psychological states, and the social desirability of the reported behavior (Katschnig, 2006). Neuroimaging shows promise as a methodology that could circumvent many of these measurement hurdles but is still in early development (Costa e Silva, 2013). Direct measurement of brain structures and networks would increase the repeatability of measures and reduce the human bias in collection. Continual or regular neuroimaging then becomes the challenge. Personal measurement devices would a possible answer to this challenge.

Markowetz and his collaborators propose the use of actigraphs for measurement and collection of psychological symptoms. Actigraphs have been used as a non-invasive method for monitoring rest and activity with measurements taken from actimerty sensors worn on the bodies of patients (Ancoli-Israel et al., 2003; Sadeh \& Acebo, 2002; Sadeh, 2011). These initial units were however still requiring substantial effort by the patient due to the sensors expense, complexity and social awkwardness. Technological advancement has 
improved these devices in recent years reducing the impracticality of clinical use. Effort by the patient is still required however due to the necessity of the sensor to be on the body of the patient for the requisite period of time. The ultimate goal here would be for the automation of this actigraphic data collection through sensor-less tracking. Connecting measurements taken about patient's brains with the continual, sensor-less tracking would facilitate the translation of neuroscience into psychology. Alterations from one neuroimaging session to the next could be associated with patterns found from the actigraph measurements.

\section{Ethical Clinical Practice}

Clinical practice is the use of scientific research and understanding to help patients with psychological malady. Evidence-based practice is shaped by novel discoveries and influence the daily activities of clinicians. Thus, maintaining an ethical clinical practice includes maintaining an adequate continuing education and professional accreditation including keeping current with the scientific discoveries shaping evidence-based practice (Dean, 2010). A self-critical eye is also important for maintenance of a professional standard of care. Having in place self-review processes allows for professionals to determine their own state of affairs and how this is affecting their clinical practice. The ever growing body of scientific knowledge coupled with broadening diversity in patient populations is where an individual clinician begins to find obstacles due to the growth in complexity of effective diagnosis and treatment (Galanti, 2001). Maintaining a current understanding of implementing best practices is shifting towards a time commitment hindering the number patients a clinician is able to provide suitable care for. Developing an adequate competency across the breadth of clinicians is the driving force behind all of the education and regulation of clinical professionals but the depth and breadth of competency is changing due to advancement of scientific research (Su-yan, 2010). As more research is created, greater understanding of both the underlying biological mechanisms as well as the treatments to remediate these disease states is generated. It could be said the boundaries of professional competency are now verging on a size too large for any one individual clinician to handle.

A team-based approach is being emphasized and is gaining greater headway in educational programs. This approach looks to capitalize on the collective efforts of the team. With the correct balance, the emergent issues present in mental healthcare would be tackled with emergent solutions stemming from effective healthcare teams. There still needs to be a greater focus put upon not only translational research but 
development of translational science focused intellectual and professional networks (Wilson-Stronks, Lee, Cordero, Kopp, \& Galvez, 2008). These additions would strengthen both individual clinicians practice as well as create integrated teams of practitioners together more capable of handling the complexities present in providing excellent healthcare. Documentation of developments made by individual practitioners and their associated teams would both allow for monitoring of effectiveness and ethical concerns.

The betterment of healthcare teams would be able to generate nuanced approaches able to tackle growing healthcare issues and provide services to a greater number of patients while still maintaining ethical standards. This increase in the effectiveness of healthcare teams would come from ethical monitoring that is able to develop with the dynamic systems of both providers and patients. Managing and analyzing for ethical concerns from the translation of science would be complimented by the use of systems science models. These models, if developed for specific teams, would have intellectual frameworks for use in clinical practice and information management. Integration of these frameworks into clinical psychologist's education, both graduate and continuing, will be where the important foundational changes will come for the improvement of competency in future clinical psychologists.

\section{Conclusions}

There is an observed trend that the growth in information available to clinicians and researchers alike, has begun to challenge these professionals to handle greater diversity and greater demands without dropping their standards. When looking to improve upon their current methods, professionals have begun to incorporate understanding and resources commonly used by other disciplines. For clinical psychologists, translation of neuroscience into their practice has shown enhanced capacity for the effective interpretation of human behavior. This clinician-level translation however begins to alter the requirements for the maintenance of evidence-based practices. Education for both current and budding professionals involved in the development of clinical psychology is a central part of maintaining the standards of care necessary for providing ethically sounds mental health services to patients. Providing resources enabling the ethical translation of neuroscience into clinical psychology then falls upon the leaders of translational science in psychology and neuroscience. Integration of basic, applied and systems neuroscience research approaches into centralized translational models is in need. The determination of useful crossover between disciplines such as neuropsychology, behavioral neuroscience 
and brain mapping projects would then facilitate the development of a psychological clinician into a brainsavvy psychological clinician. Clinicians with translational abilities would then be able to integrate developments in neuroscience, including that of neuroimaging, brain biomarkers and personal measurement devices, into clinical practice. Measurements of neuroplasticity changes in the brain as well as having the ability to monitor behavioral patterns overtime, outside the clinic, opens a window into brain and psychological understandings that will lead to fundamental shifts in research and clinical practice.

A number of academic journals and books have focused on translational applications of neuroscience in clinical psychology. The resource focuses follow similar field distinctions as outlined looking to bring the different research approaches and professional disciplines together and making them available to interested clinicians. Four journals with prevalent publication numbers on the translation of neuroscience are NeuroImage, Behavioral and Brain Sciences, Frontiers in Systems Neuroscience and Translational Neuroscience. A book that emphasizes the translation of neuroscience into clinical psychology is Neuroscience in Psychotherapy by Louis Cozolino. These mediums develop the useful intersections between basic and applied sciences as well as neuroscience and psychology. Promotion and use of these, and similar, resources will continue to improve the availability of materials and tools useful in the translation of neuroscience into clinical psychology.

Considerations for future research are a deeper ethical reflection on clinician translation of neuroscience science in psychological practice as well as the financial and practical hurdles present for the development of professional crowd sourced translational resources. Effective treatment of psychological disease and illness will gain much from the integration of dynamic and translational models. Increased collaboration of clinicians and researchers across professional disciplines and research fields will certainly promote the increase in complexity for understanding these problems. What is most important to consider however, is the enhanced ability for humans to take on the unknown complexities of the human brain. 


\section{Citations}

American Psychological Association. (2006). Evidence-based practice in psychology. American Psychologist, 61(4), 271-285. doi:10.1037/0003-066X.61.4.271

Ancoli-Israel, S., Cole, R., Alessi, C., Chambers, M., Moorcroft, W., \& Pollak, C. P. (2003). The role of actigraphy in the study of sleep and circadian rhythms. Sleep, 26(3), 342-392.

Assaf, Y., Alexander, D. C., Jones, D. K., Bizzi, A., Behrens, T. E. J., Clark, C. A., .. Poupon, C. (2013). The CONNECT project: Combining macro- and micro-structure. NeuroImage, 80, 273-282. doi:10.1016/j.neuroimage.2013.05.055

Atkins, M. S., Strauman, T. J., Cyranowski, J. M., \& Kolden, G. G. (2014). Reconceptualizing Internship Training Within the Evolving Clinical Science Training Model. Clinical Psychological Science, 2(1), 46-57. doi:10.1177/2167702613501308

Atkinson, A. J., Colburn, W. A., DeGruttola, V. G., DeMets, D. L., Downing, G. J., Hoth, D. F., ... Zeger, S. L. (2001). Biomarkers and surrogate endpoints: Preferred definitions and conceptual framework*. Clinical Pharmacology \& Therapeutics, 69(3), 89-95. doi:10.1067/mcp.2001.113989

Biswal, B. B., Mennes, M., Zuo, X.-N., Gohel, S., Kelly, C., Smith, S. M., ... Milham, M. P. (2010). Toward discovery science of human brain function. Proceedings of the National Academy of Sciences, 107(10), 4734-4739. doi:10.1073/pnas.0911855107

Braff L, \& Braff DL. (2013). The neuropsychiatric translational revolution: Still very early and still very challenging. JAMA Psychiatry, 70(8), 777-779. doi:10.1001/jamapsychiatry.2013.2184

Castellanos, F. X., Di Martino, A., Craddock, R. C., Mehta, A. D., \& Milham, M. P. (2013). Clinical applications of the functional connectome. NeuroImage, 80, 527-540. doi:10.1016/j.neuroimage.2013.04.083

Costa e Silva, J. A. (2013). Personalized medicine in psychiatry: New technologies and approaches. Metabolism, 62, S40-S44. doi:10.1016/j.metabol.2012.08.017

Coverdale, J., Balon, R., Beresin, E. V., Louie, A. K., Tait, G. R., Goldsmith, M., \& Roberts, L. W. (2014). Teaching Clinical Neuroscience to Psychiatry Residents: Model Curricula. Academic Psychiatry, 38(2), 111-115. doi:10.1007/s40596-014-0045-7

Dean, D. M. (2010). Clinical Competency and Ethics in Psychology. Student Pulse, 2(10). Retrieved from http://www.studentpulse.com/a?id=308 
Galanti, G.-A. (2001). The challenge of serving and working with diverse populations in American hospitals. Diversity Factor, 9(3), 21-26.

Green, L. W. (2006). Public Health Asks of Systems Science: To Advance Our Evidence-Based Practice, Can You Help Us Get More Practice-Based Evidence? American Journal of Public Health, 96(3), 406-409. doi:10.2105/AJPH.2005.066035

Gyani, A., Shafran, R., Myles, P., \& Rose, S. (2014). The Gap Between Science and Practice: How Therapists Make Their Clinical Decisions. Behavior Therapy, 45(2), 199-211. doi:10.1016/j.beth.2013.10.004

Harriette, J. (2014). Behavioral Neuroscience for the Human Services: Foundations in Emotion, Mental Health, Addiction, and Alternative Therapies (1 edition.). Oxford: Oxford University Press.

Harvey, P. D. (2012). Clinical applications of neuropsychological assessment. Dialogues in Clinical Neuroscience, 14(1), 91-99.

Hieronymi, A. (2013). Understanding Systems Science: A Visual and Integrative Approach. Systems Research and Behavioral Science, 30(5), 580-595. doi:10.1002/sres.2215

Jiang, T. (2013). Brainnetome: A new -ome to understand the brain and its disorders. Neuroimage, 80, 263-272. doi:10.1016/j.neuroimage.2013.04.002

Katschnig, H. (2006). Quality of life in mental disorders: challenges for research and clinical practice. World Psychiatry, 5(3), 139-145.

Kelly, A. M. C. (2004). Human Functional Neuroimaging of Brain Changes Associated with Practice. Cerebral Cortex, 15(8), 1089-1102. doi:10.1093/cercor/bhi005

Kelly, C., \& Castellanos, F. X. (2014). Strengthening Connections: Functional Connectivity and Brain Plasticity. Neuropsychology Review, 24(1), 63-76. doi:10.1007/s11065-014-9252-y

Postgrad growth area: Translational science. (n.d.). http://www.apa.org. Retrieved May 19, 2014, from http://www.apa.org/gradpsych/2011/01/postgrad.aspx

Sadeh, A. (2011). The role and validity of actigraphy in sleep medicine: An update. Sleep Medicine Reviews, 15(4), 259-267. doi:10.1016/j.smrv.2010.10.001

Sadeh, A., \& Acebo, C. (2002). The role of actigraphy in sleep medicine. Sleep Medicine Reviews, 6(2), 113-124. doi:10.1053/smrv.2001.0182 
Shoham, V., Rohrbaugh, M. J., Onken, L. S., Cuthbert, B. N., Beveridge, R. M., \& Fowles, T. R. (2014). Redefining Clinical Science Training Purpose and Products of the Delaware Project. Clinical Psychological Science, 2(1), 8-21. doi:10.1177/2167702613497931

Steen, R. G., Casadevall, A., \& Fang, F. C. (2013). Why Has the Number of Scientific Retractions Increased? PLoS ONE, 8(7), e68397. doi:10.1371/journal.pone.0068397

Su-yan, L. B. (2010). Treating Patients from Diverse Populations, 30-33.

Thyer, B. A., \& Myers, L. L. (2011). The quest for evidence-based practice: A view from the United States. Journal of Social Work, 11(1), 8-25. doi:10.1177/1468017310381812

Translational Psychology. (n.d.). http://www.apa.org. Retrieved May 19, 2014, from http://www.apa.org/science/about/psa/2007/07/ed-column.aspx

Van Essen, D. C., Smith, S. M., Barch, D. M., Behrens, T. E. J., Yacoub, E., \& Ugurbil, K. (2013). The WU-Minn Human Connectome Project: An overview. NeuroImage, 80, 62-79. doi:10.1016/j.neuroimage.2013.05.041

Wilson-Stronks, A., Lee, K., Cordero, C., Kopp, A., \& Galvez, E. (2008). HLCOneSizeFinal.pdf. The Join Commisisinon.

Woolf SH. (2008). The meaning of translational research and why it matters. JAMA, 299(2), 211-213. doi:10.1001/jama.2007.26

Woolrich, M. W., \& Stephan, K. E. (2013). Biophysical network models and the human connectome. NeuroImage, 80, 330-338. doi:10.1016/j.neuroimage.2013.03.059

Yan, C.-G., Craddock, R. C., Zuo, X.-N., Zang, Y.-F., \& Milham, M. P. (2013). Standardizing the intrinsic brain: Towards robust measurement of inter-individual variation in 1000 functional connectomes. NeuroImage, 80, 246-262. doi:10.1016/j.neuroimage.2013.04.081

Zerhouni, E. A. (2005). Translational and Clinical Science — Time for a New Vision. New England Journal of Medicine, 353(15), 1621-1623. doi:10.1056/NEJMsb053723 